TRANSACTIONS OF THE

AMERICAN MATHEMATICAL SOCIETY

Volume 359, Number 7, July 2007, Pages 3483-3503

S 0002-9947(07)04147-5

Article electronically published on February 13, 2007

\title{
ANALOGUE OF THE DEGREE CONJECTURE OVER FUNCTION FIELDS
}

\author{
MIHRAN PAPIKIAN
}

\begin{abstract}
Under a certain assumption, similar to Manin's conjecture, we prove an upper bound on the degree of modular parametrizations of elliptic curves by Drinfeld modular curves, which is the function field analogue of the conjectured bound over the rational numbers.
\end{abstract}

\section{INTRODUCTION}

Let $E$ be a semi-stable elliptic curve over $\mathbb{Q}$ of conductor $N_{E}, \mathcal{H}$ be the complex upper half-plane, and $\Gamma_{0}\left(N_{E}\right)$ be the Hecke congruence subgroup of level $N_{E}$. Denote the compactification of the quotient $\Gamma_{0}\left(N_{E}\right) \backslash \mathcal{H}$ by $X_{0}\left(N_{E}\right)$. One can view $X_{0}\left(N_{E}\right)$ as an algebraic curve over $\mathbb{C}$ (and in fact over $\mathbb{Q}$ ). From the work of Wiles and Taylor it is known that there is a non-constant map

$$
\wp: X_{0}\left(N_{E}\right) \rightarrow E
$$

called modular parametrization. We always choose a modular parametrization of minimal degree. Assume further that $E$ is optimal, that is, the map induced by $\wp$ between the Albanese varieties has a connected (and smooth) kernel. It is known that every $\mathbb{Q}$-isogeny class of elliptic curves contains a unique such curve. The optimal curve in a given $\mathbb{Q}$-isogeny class can also be characterized as the curve for which $\operatorname{deg} \wp$ is minimal. G. Frey made the following remarkable conjecture:

Conjecture 1.1 (Degree conjecture). For any $\varepsilon>0$ there is a constant $c(\varepsilon)$ depending only on $\varepsilon$ such that

$$
\operatorname{deg} \wp \leq c(\varepsilon) N_{E}^{2+\varepsilon} .
$$

This conjecture is known to imply the celebrated $A B C$ conjecture, and also implies a bound on congruence primes of the newform corresponding to $E$; see [13], [15]. In this paper, under a certain assumption, we will prove the function field analogue of this conjecture.

Let $\mathbb{F}_{q}$ be the finite field of $q$ elements, and let $C / \mathbb{F}_{q}$ be a smooth, projective, geometrically irreducible curve over $\mathbb{F}_{q}$. Denote by $F$ the function field of $C$, and by $A$ the subring of $F$ consisting of functions which are regular outside a fixed place

Received by the editors October 27, 2004 and, in revised form, July 26, 2005.

2000 Mathematics Subject Classification. Primary 11G05; Secondary 11G18.

Key words and phrases. Degree conjecture, Drinfeld modular curves, ABC conjecture, monodromy pairing.

This research was supported by a fellowship from the European Postdoctoral Institute.

(C)2007 American Mathematical Society

Reverts to public domain 28 years from publication 
$\infty$ on $C$. ( $F$ and $A$ are the analogues of $\mathbb{Q}$ and $\mathbb{Z}$ respectively.) In this setting the role of the complex upper half-plane is played by the Drinfeld upper half-plane

$$
\Omega=\widehat{\bar{F}}_{\infty}-F_{\infty},
$$

where $F_{\infty}$ is the completion of $F$ at $\infty$, and $\widehat{\bar{F}}_{\infty}$ is the completion of the algebraic closure of $F_{\infty}$. The upper half-plane $\Omega$ can be endowed with a structure of a one-dimensional rigid-analytic space. Fix an ideal $\mathfrak{n}$ of $A$ and denote by $\Gamma_{0}(\mathfrak{n})$ the subgroup of $\mathrm{GL}_{2}(A)$ whose image in $\mathrm{GL}_{2}(A / \mathfrak{n})$ consists of upper-triangular matrices. The subgroup $\Gamma_{0}(\mathfrak{n})$ acts on $\Omega$ through linear fractional transformations. Drinfeld showed that the quotient $\Gamma_{0}(\mathfrak{n}) \backslash \Omega$ is the underlying rigid-analytic space of an affine algebraic curve defined over $F_{\infty}$. The compactification $X_{0}(\mathfrak{n})$ of this curve is called the Drinfeld modular curve for $\Gamma_{0}(\mathfrak{n})$. The analogue of the modularity conjecture over function fields is a consequence of some deep results of Deligne, Drinfeld and Zarhin, cf. [9]: Let $E$ be an elliptic curve over $F$ with conductor $\mathfrak{n}_{E}=\mathfrak{n} \cdot \infty$ and split multiplicative reduction at $\infty$. Then there is a non-constant morphism

$$
\wp: X_{0}(\mathfrak{n}) \rightarrow E_{/ F_{\infty}} .
$$

In fact this map can be defined over certain finite Galois extension of $F$. As over $\mathbb{Q}$, we choose $\wp$ of minimal degree. Let $\mathcal{T}$ denote the Bruhat-Tits tree of $\mathrm{PGL}_{2}\left(F_{\infty}\right)$. The group $\Gamma:=\Gamma_{0}(\mathfrak{n})$ naturally acts on $\mathcal{T}$, and the homology group $H_{1}(\Gamma \backslash \mathcal{T}, \mathbb{Z})$ of the quotient graph is free of $\mathbb{Z}$-rank equal to the genus of $X_{0}(\mathfrak{n})$. There is a natural injective homomorphism $\iota: \mathrm{H}_{1}(\Gamma \backslash \mathcal{T}, \mathbb{Z}) \rightarrow \underline{\mathrm{H}}_{!}(\mathcal{T}, \mathbb{C})^{\Gamma}$, where $\underline{\mathrm{H}}_{!}(\mathcal{T}, \mathbb{C})^{\Gamma}$ is the vector space of $\mathbb{C}$-valued Drinfeld cusp forms for $\Gamma$ (also called harmonic cochains); see $[9, \S 3]$. The space $\underline{H}_{!}(\mathcal{T}, \mathbb{C})^{\Gamma}$ plays a role similar to that of weight-2 cusp forms of a given level in the classical theory. One can associate to our elliptic curve $E$ a primitive element $v_{E} \in \mathrm{H}_{1}(\Gamma \backslash \mathcal{T}, \mathbb{Z}$ ), well-defined up to a sign. (Primitive means that $v_{E}$ is not an integer multiple of any other element except $-v_{E}$.) We make the assumption $(*)$ that the element $\iota\left(v_{E}\right)$ is the normalized newform corresponding to $E$. Also assume $E$ is semi-stable and optimal. The main result of this paper is the following bound:

Theorem 1.2. With previous notation and assumptions, we have

$$
\operatorname{deg} \wp<q^{(14 g+5+\operatorname{deg} \infty)} \cdot \mathcal{N}\left(\mathfrak{n}_{E}\right) \cdot\left(\operatorname{deg} \mathfrak{n}_{E}\right)^{3},
$$

where $\mathcal{N}\left(\mathfrak{n}_{E}\right):=q^{\operatorname{deg} \mathfrak{n}_{E}}, g$ is the genus of the curve $C$ and $\operatorname{deg}$ denotes the degree of Weil divisors on $C$.

Taking $g$ and $\operatorname{deg}(\infty)$ as the fixed data attached to $F$ and $A$, we see that the bound in the theorem is somewhat better than the conjectured bound over $\mathbb{Q}$, as it is analogous to the upper bound $N_{E}\left(\log N_{E}\right)^{3}$. The degree of optimal modular parametrization over function fields can be shown to be divisible by the congruence primes of $E$; see [7. Hence our theorem proves the analogue of Murty's conjecture [15. Conj. D] (modulo the assumptions we made). Using the argument in the proof of [17, Thm.6.4], one can show that $\operatorname{deg} \wp$ is bounded from below by $\alpha$. $\mathcal{N}\left(\mathfrak{n}_{E}\right) /(\operatorname{deg} \mathfrak{n})^{2}$, where $\alpha$ is a constant depending only on $q, g$ and $\operatorname{deg} \infty$. Thus, $\log (\operatorname{deg} \wp) \asymp \operatorname{deg} \mathfrak{n}_{E}$ and the bound in the theorem is essentially the best possible.

We make a few comments on our assumptions. The assumption that $E$ is semistable is not essential. The only reason why we made this assumption is to avoid some technical nuances in the analytic calculations in 84, see Remark 4.3. On 
the other hand, the assumption that $E$ is optimal cannot be discarded. Over $\mathbb{Q}$ the degree conjecture can be stated without assuming the elliptic curve is optimal. This is equivalent to the original conjecture, since by Mazur's fundamental result any two elliptic curves in the same $\mathbb{Q}$-isogeny class can be related by an isogeny of degree at most 163. Due to the existence of the Frobenius morphism, this is not the case over function fields. In fact, any isogeny class of non-isotrivial elliptic curves over $F$ contains infinitely many curves, and the degrees of minimal possible isogenies between them can be arbitrarily large. Hence to ask a meaningful question on how large $\operatorname{deg} \wp$ can be, one has to restrict to optimal curves. Finally about $(*)$. In the statement of Conjecture 1.1 one implicitly uses the results of Mazur and Raynaud which say that for semi-stable elliptic curves Manin's constant $c_{E}$ is 1 or 2 (and Manin conjectures that $c_{E}$ is always equal to 1). Otherwise the bound in the conjecture has to be multiplied by $c_{E}^{2}$; see [13]. Our assumption $(*)$ is of similar nature. If $\iota\left(v_{E}\right)$ is a $\widetilde{c}_{E}$ multiple of the newform corresponding to $E$, then we can drop $(*)$ by multiplying the right-hand side of the inequality in Theorem 1.2 by $\left(\widetilde{c}_{E}\right)^{2}$. In both cases the reason for the appearance of the constant $c^{2}$ is that the degree of optimal modular parametrization is proportional to the Petersson norm of $c \cdot f_{E}$, with $f_{E}$ being the normalized newform associated to $E$. In fact, the proof of Theorem 1.2 proceeds by first establishing the following formula for $\operatorname{deg} \wp$, which might be of some independent interest:

Proposition 1.3. Assume $E$ is optimal. Then

$$
\operatorname{deg} \wp=q^{2 g-2} \mathcal{N}\left(\mathfrak{n}_{E}\right) \cdot \frac{\left(\widetilde{c}_{E}\right)^{2} \cdot\left(\# \operatorname{coker} \wp_{*}^{\Phi}\right)^{2}}{\# \Phi_{E, \infty}} \cdot L\left(1, \operatorname{Sym}^{2} \pi_{E}\right) .
$$

Here $\Phi_{E, \infty}$ is the group of connected components of the Néron model of $E$ over $\infty$, $\wp_{*}^{\Phi}$ is the functorial homomorphism $\Phi_{J, \infty} \rightarrow \Phi_{E, \infty}$ between the component groups of Néron models of the Jacobian of $X_{0}(\mathfrak{n})$ and $E$ induced by $\wp$, and $\pi_{E}$ is the automorphic cuspidal representation attached to $E$.

It can be shown that $\widetilde{c}_{E} \in \mathbb{Z}$, and it is natural to expect that $\widetilde{c}_{E}=1$; cf. 9 , (6.4.4)]. We note that Proposition 1.3 generalizes the formula [18, (27)] for the case of $\mathbb{F}_{q}(t)$ to general function fields.

The main obstacle in proving the degree conjecture over $\mathbb{Q}$ lies in giving lower bounds on the area of the fundamental parallelogram of the lattice of periods of $E, \int_{E(\mathbb{C})}\left|\omega_{E}\right|$, in terms of the conductor of $E$; cf. [15]. (Here $\omega_{E}$ is a Néron differential of $E$.) Over function fields this quantity gets replaced by $\# \Phi_{E, \infty}$, and hence obviously is larger than or equal to 1 . This trivial lower bound on $\# \Phi_{E, \infty}$ turns out to be sufficient to deduce the bound in Theorem [1.2. Note that by a theorem of Tate $\# \Phi_{E, \infty}$ is essentially $\int_{E\left(F_{\infty}\right)}\left|\omega_{E}\right|$, hence one could say that the degree conjecture is possible to prove over function fields because a certain complex period gets replaced by a non-archimedean period.

The challenge in proving Theorem 1.2 is primarily in relating $\operatorname{deg} \wp$ to the Petersson norm of $f_{E}$. Over $\mathbb{C}$ one derives the analogous formula by pulling back via $\wp$ a holomorphic differential form on $E$ to $X_{0}\left(N_{E}\right)$. This approach does not work over $F$ since it has positive characteristic and we essentially see $\operatorname{deg} \wp$ only modulo the characteristic. The remedy consists of looking at the induced maps between integral homology groups (or rather their analogues in the rigid-analytic setting). The idea of doing this is due to Gekeler [7. We used his results to prove in [18] a slightly weaker version of Theorem 1.2 for the case of $A=\mathbb{F}_{q}[t]$. In this paper 
we deal with general function fields by using Grothendieck's monodromy pairing instead, which seems better adapted to this situation.

The organization of the paper is as follows: Section 2 is algebraic in nature and gives a formula for $\operatorname{deg} \wp$ in terms of Grothendieck's monodromy pairing. Section 3 contains the proof of the main theorem, Theorem 1.2. We relegated all analytic calculations required in the proof of the main theorem to the last section, Section 4. This consists of computing certain Rankin-Selberg integrals and bounding the special values of symmetric power $L$-functions for GL(2).

\section{Monodromy PAIRING AND A FORMUla FOR DEGREe}

In this section we let $R$ be a complete discrete valuation ring, $K$ be its field of fractions, and $k$ be the residue field. By a finite flat group scheme over the base scheme $S$ we always mean a finite flat commutative $S$-group scheme. When $S=\operatorname{Spec}(L)$ with $L$ a field, we will abbreviate this to "finite $L$-group scheme". If $G$ is a finite flat group scheme over a connected base $S$, then the $\mathcal{O}_{S}$-module $\mathcal{O}_{G}$ is locally free of constant rank called the order of $G$ and denoted $\# G$. A finite group scheme $G$ over a field $L$ is said to be étale if $G \times{ }_{\operatorname{Spec}(L)} \operatorname{Spec}(\bar{L})$ is reduced, where $\bar{L}$ is the algebraic closure of $L$. As is well-known, there is a one-to-one correspondence between the finite étale group schemes over $L$ and the finite abelian groups with a continuous action of $\operatorname{Gal}\left(L^{\mathrm{sep}} / L\right)$. It is also well-known that if $G$ over $L$ has order coprime to the characteristic of $L$, then $G$ is étale. A finite flat group scheme $G$ over $S$ is said to be étale if the fibres $G_{s}$ are étale over the corresponding residue fields for all closed points $s$ of $S$. We say that a finite flat group scheme $G$ is multiplicative if its Cartier dual $G^{\vee}$ is étale.

2.1. Néron models of abelian varieties with toric reduction. Let $A$ be an abelian variety over $K$. Denote by $\mathcal{A}$ its Néron model over $R$ and denote by $\mathcal{A}_{k}^{0}$ the connected component of the identity of the closed fibre $\mathcal{A}_{k}$ of $\mathcal{A}$. We have an exact sequence

$$
0 \rightarrow \mathcal{A}_{k}^{0} \rightarrow \mathcal{A}_{k} \rightarrow \Phi_{A} \rightarrow 0,
$$

where $\Phi_{A}$ is a finite étale group scheme over $k$ called the component group of $A$. We say that $A$ has (split) toric reduction if $\mathcal{A}_{k}^{0}$ is a (split) torus.

In general, the closed immersions of abelian varieties do not extend to closed immersions of Néron models; see [1, §7.5]. Nevertheless, under special assumptions this is true; cf. [1, Thm.7.5/4], where $R$ is assumed to be of mixed characteristic with a "small" absolute ramification index. In Proposition 2.3 we will prove a similar (slightly weaker) statement for equicharacteristic $R$, assuming the abelian varieties in question have toric reduction. We replace the use of Raynaud's theorem [21, Cor. 3.3.6], which is valid only in mixed characteristic, by Lemma 2.1.

Let $G$ be a finite $K$-group scheme, and let $\mathcal{G}$ be a finite flat $R$-group scheme extending $G$ (i.e., $\mathcal{G}_{K}=G$ ). We say that $\mathcal{G}$ is an admissible extension of $G$ if the connected component $\mathcal{G}_{k}^{0}$ of the closed fibre of $\mathcal{G}$ is multiplicative (we allow $\mathcal{G}_{k}^{0}$ to be trivial).

Lemma 2.1. Assume $R$ is equicharacteristic. Let $G$ be a finite group scheme over $K$ of order equal to a power of a prime number $p$. Let $\mathcal{G}^{\prime}$ and $\mathcal{G}^{\prime \prime}$ be two admissible extensions of $G$. Any map $v: \mathcal{G}^{\prime} \rightarrow \mathcal{G}^{\prime \prime}$ which is an isomorphism over $K$ is an isomorphism over $R$. 
Proof. If $p$ is not the characteristic of $K$, then any finite flat extension of $G$ over $R$ must be étale and is clearly unique, so we will assume $p$ is the characteristic of $K$. Let $\mathcal{G}$ be any admissible extension. Consider the connected-étale sequence of $\mathcal{G}$

$$
0 \rightarrow \mathcal{G}^{0} \rightarrow \mathcal{G} \rightarrow \mathcal{G}^{\text {et }} \rightarrow 0 .
$$

The formation of this sequence is compatible with any local base change to another henselian local ring (e.g., passage to the closed fibre $R \rightarrow k$ ), so

$$
0 \rightarrow \mathcal{G}_{k}^{0} \rightarrow \mathcal{G}_{k} \rightarrow \mathcal{G}_{k}^{\text {et }} \rightarrow 0
$$

is the connected-étale sequence of $\mathcal{G}_{k}$. Next, we claim that the generic fibre $\mathcal{G}_{K}^{0}$ of $\mathcal{G}^{0}$ must be multiplicative. It is enough to show that the Cartier dual $\mathcal{H}$ of $\mathcal{G}^{0}$ has étale $K$-fibre. Since by assumption $\mathcal{G}_{k}^{0}$ is multiplicative, $\mathcal{H}_{k}$ is étale. This implies that $\mathcal{H}$ is étale, and in particular $\mathcal{H}_{K}$ is étale. Multiplicative $p$-power order group schemes are connected in characteristic $p$, so $\mathcal{G}_{K}^{0}$ is a subgroup scheme of $G^{0}$. Since $\left(\mathcal{G}^{\mathrm{et}}\right)_{K}$ is étale, we see that

$$
0 \rightarrow \mathcal{G}_{K}^{0} \rightarrow \mathcal{G}_{K} \rightarrow \mathcal{G}_{K}^{\mathrm{et}} \rightarrow 0
$$

is the connected-étale sequence of $G$. (Note that the formation of the connectedétale sequence usually does not commute with non-local base change, such as $R \rightarrow$ $K$, so for our last conclusion the admissibility of $\mathcal{G}$ is crucial.)

Now let $\mathcal{G}^{\prime}$ and $\mathcal{G}^{\prime \prime}$ be two admissible extensions of $G$. Let $v: \mathcal{G}^{\prime} \rightarrow \mathcal{G}^{\prime \prime}$ be a morphism over $R$ which is an isomorphism over $K$. There are unique maps $v^{0}$ and $v^{\text {et }}$ between connected components and maximal étale quotients such that the diagram

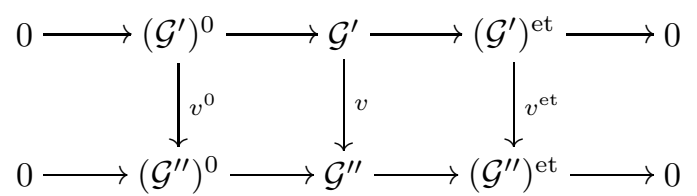

commutes. From what we proved we conclude that $\left(\mathcal{G}^{\prime}\right)^{0}$ and $\left(\mathcal{G}^{\prime \prime}\right)^{0}$ are two multiplicative extensions of the same multiplicative $K$-group scheme $G^{0}$. Since $G^{0}$ has a unique such extension (for example, take the Cartier duals to get étale group schemes where this is obvious), $v^{0}$ is an isomorphism. Next, $\left(\mathcal{G}^{\prime}\right)^{\text {et }}$ and $\left(\mathcal{G}^{\prime \prime}\right)^{\text {et }}$ are étale extensions of $G^{\text {et }}$, hence $v^{\text {et }}$ is also an isomorphism. We conclude that $v$ is an isomorphism.

Remark 2.2.

(1) The statement of the lemma is false without assuming $R$ is equicharacteristic, as the example of $\mu_{2}$ over $\mathbb{Q}_{2}$ shows.

(2) When the order of $G$ is coprime to the characteristic of $R$, then it extends over $R$ if and only if $G(\bar{K})$ is unramified.

(3) When $R$ has characteristic $p>0, G$ need not have any admissible extensions over $R$ even if it is étale or multiplicative of order $p$. Take for example the Oort-Tate group scheme $G_{a, 0}, a \in K$ with $0<\operatorname{ord}_{K}(a)<p-1$; see [16]. This is étale, but any extension $\mathcal{G}$ has a closed fibre isomorphic to $G_{0,0 / k}=\alpha_{p}$. Also, there are commutative group schemes over $K$ killed by $p$ and of order $p^{2}$ which do not extend over $R$ at all; see Appendix B of Ch.3 in [14. 
Proposition 2.3. Let $f_{K}: A \rightarrow B$ be a closed immersion of abelian varieties over $K$, i.e., $A$ is an abelian subvariety of $B$. Assume $B$ has toric reduction. If $R$ is equicharacteristic, then the canonical morphism $f: \mathcal{A} \rightarrow \mathcal{B}$ extending $f_{K}$ induces a closed immersion $f_{k}: \mathcal{A}_{k} \rightarrow \mathcal{B}_{k}$.

Proof. If the characteristic of $R$ is zero, then in fact a stronger statement is true, cf. [1, Prop.7.5/2], so we will assume $R$ has positive characteristic $p$.

By slightly modifying the argument in [1, Lem.7.4/2], one can show that $A$ also has toric reduction. Since a monomorphism between smooth finite type group schemes over a field is necessarily a closed immersion, it suffices to show that $H:=\operatorname{ker} f_{k}$ is trivial. The kernel of $f_{k}: \mathcal{A}_{k} \rightarrow \mathcal{B}_{k}$ is finite, as $A$ is isogenous (in fact isomorphic) to a subvariety of $B$. Hence $H$ is finite, and if we show that it has trivial $\ell$-torsion for any prime $\ell$, we could conclude that $H$ is trivial. As $H[\ell] \subseteq \mathcal{A}[\ell]_{k}$, it is enough to show that $f$ restricted to the quasi-finite and flat group scheme $\mathcal{A}[\ell]$ is a monomorphism. Since $R$ is henselian, there is a unique decomposition $\mathcal{A}[\ell]=G \amalg G_{0}$ into disjoint subgroup schemes which are both open and closed in $\mathcal{A}[\ell]$, and such that $G$ is finite and flat over $R$ and $G_{0}$ has empty closed fibre; cf. 1 , $\S 7.3]$. Hence it is enough to prove that $f$ is an isomorphism when restricted to $G$. Let $G^{\prime}$ be the schematic image of $G$ in $\mathcal{B}$ under $f$. Since $f_{K}$ is a closed immersion, we have an isomorphism on generic fibres $f_{K}: G_{K} \stackrel{\sim}{\longrightarrow} G_{K}^{\prime}$. Since $A$ has toric reduction, $G_{k}^{0}$ is multiplicative (being in the kernel of a homomorphism of tori). Similarly for $G^{\prime}$. We conclude that $G$ and $G^{\prime}$ are two admissible extensions of $G_{K}$, and $f$ must be an isomorphism by Lemma 2.1.

Let $A$ and $B$ be as in Proposition 2.3 . Consider the commutative diagram induced by $f_{k}$ :

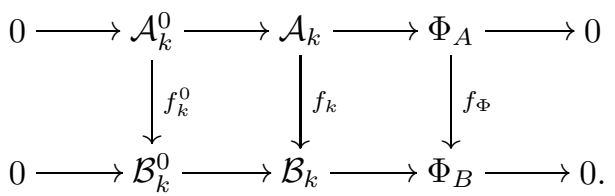

Corollary 2.4. The morphism $f_{k}^{0}$ is a closed immersion and $\operatorname{ker}\left(f_{\Phi}\right)$ can be identified with a $\operatorname{Gal}(\bar{k} / k)$-subgroup of the quotient torus $\mathcal{B}_{k}^{0} / \mathcal{A}_{k}^{0}$.

Proof. Follows immediately from Proposition 2.3 by using the snake lemma.

2.2. Monodromy pairing and component groups. Let $A$ be an abelian variety over $K$ with toric reduction. Let $A^{\vee}$ be the dual abelian variety of $A$. The character group

$$
M_{A}=\operatorname{Hom}_{\bar{k}}\left(\mathcal{A}_{\bar{k}}^{0}, \mathbb{G}_{m, \bar{k}}\right)
$$

is a free abelian group contravariantly associated to $A$; its $\mathbb{Z}$-rank is equal to the dimension of $A$. Let $M_{A^{\vee}}$ be the analogous group associated to $A^{\vee}$. In [11, §§9-10] Grothendieck defined a canonical bilinear $\operatorname{Gal}(\bar{k} / k)$-equivariant pairing,

$$
u_{A}: M_{A} \times M_{A} \vee \rightarrow \mathbb{Z},
$$

which he called the monodromy pairing. This pairing is uniquely characterized by the property that its extension of scalars $u_{A} \otimes \mathbb{Z}_{\ell}$, for a prime $\ell \neq \operatorname{char}(k)$, can be expressed in terms of the $\ell$-adic Weil pairing on $T_{\ell}(A) \times T_{\ell}\left(A^{\vee}\right)$ via a formula given in [11, (9.1.2)]. The monodromy pairing has the following properties. 
Theorem 2.5 (Grothendieck).

(i) $u_{A}$ is non-degenerate.

(ii) $u_{A}$ is bifunctorial.

(iii) If $\xi: A^{\vee} \rightarrow A$ is a polarization, then

$$
u_{A, \xi}: M_{A} \times M_{A} \stackrel{1 \times \xi}{\longrightarrow} M_{A} \times M_{A} \stackrel{u_{A}}{\longrightarrow} \mathbb{Z}
$$

is symmetric and positive-definite.

(iv) There is a $\operatorname{Gal}(\bar{k} / k)$-equivariant exact sequence

$$
0 \longrightarrow M_{A^{\vee}} \stackrel{u_{A}}{\longrightarrow} \operatorname{Hom}\left(M_{A}, \mathbb{Z}\right) \longrightarrow \Phi_{A} \longrightarrow 0 .
$$

Proof. Once $u_{A}$ is constructed compatibly with the Weil pairing (this is quite nontrivial), properties (i)-(iii) follow from well-known facts about the latter pairing; see [11, Thm. 10.4]. For the proof of (iv) see [11, Thm. 11.5].

Definition 2.6. The abelian variety $A$ is an optimal quotient of the abelian variety $B$ if there is a faithfully flat morphism $\pi: B \rightarrow A$ whose functorial kernel is represented by an abelian subvariety of $B$ (that is, $\operatorname{ker} \pi$ is connected and smooth).

From now on we will assume that $R$ is equicharacteristic, so as to have Corollary 2.4 at our disposal. Let $\pi: B \rightarrow A$ be as in Definition 2.6. and assume $B$, hence also $A$, to have toric reduction. Consider the commutative diagram induced by $\pi$ on the sequence in Theorem 2.5(iv)

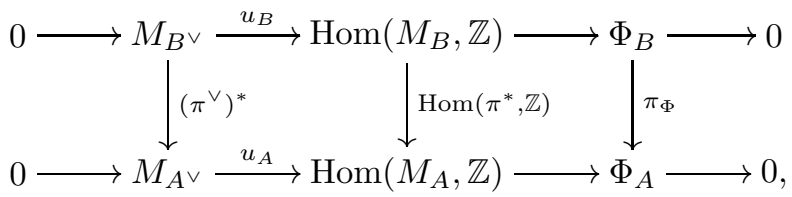

where $\pi^{\vee}: A^{\vee} \rightarrow B^{\vee}$ is the dual morphism of $\pi$, and $\pi^{*},\left(\pi^{\vee}\right)^{*}$ are the functorially induced homomorphisms between the character groups. It is a general fact that the duals of optimal quotients are closed immersions, so $\pi^{\vee}$ is a closed immersion. By Corollary 2.4, $\left(\mathcal{A}^{\vee}\right)_{k}^{0} \rightarrow\left(\mathcal{B}^{\vee}\right)_{k}^{0}$ is a closed immersion, so $\left(\pi^{\vee}\right)^{*}$ is surjective. From the snake lemma we get

$$
\# \operatorname{coker}\left(\pi_{\Phi}\right)=\# \operatorname{Ext}_{\mathbb{Z}}^{1}\left(M_{B} / \pi^{*}\left(M_{A}\right), \mathbb{Z}\right)=\#\left(M_{B} / \pi^{*}\left(M_{A}\right)\right)_{\text {tor }} .
$$

There is a bifunctorial pairing $\Phi_{A} \times \Phi_{A \vee} \rightarrow \mathbb{Q} / \mathbb{Z}$, defined by Grothendieck in [11, $\$ 1.2$ for any abelian variety $A$ over $K$, which turns out to be perfect when $A$ is semi-stable (in particular, when $A$ has toric reduction); see [11, §11.3]. Applied to the situation at hand, this pairing induces canonical isomorphisms between $\Phi_{A}$ and the Pontrjagin dual $\left(\Phi_{A^{\vee}}\right)^{*}$ of $\Phi_{A^{\vee}}$, and between $\operatorname{ker}\left(\pi_{\Phi}^{\vee}\right)$ and the Pontrjagin dual $\operatorname{coker}\left(\pi_{\Phi}\right)^{*}$. In particular, $\# \operatorname{coker}\left(\pi_{\Phi}\right)=\# \operatorname{ker}\left(\pi_{\Phi}^{\vee}\right)$.

Proposition 2.7. With notation as above, assume further that $B$ has split toric reduction. Let $q:=\# k$, and let $s$ be the minimum of $\operatorname{dim}(A)$ and $\operatorname{dim}(B)-\operatorname{dim}(A)$. Then \# $\operatorname{coker}\left(\pi_{\Phi}\right)$ divides $(q-1)^{s}$.

Proof. If $B$ has split toric reduction, then so do $A$ and $A^{\vee}$. Hence the character groups $M_{A}$ and $M_{A \vee}$ have trivial Gal $(\bar{k} / k)$-action. From Theorem 2.5(iv) we conclude that $\Phi_{A}$ and $\Phi_{A} \vee$ are constant étale group schemes over $k$ with at most $\operatorname{dim}(A)$ generators. We know that $\# \operatorname{coker}\left(\pi_{\Phi}\right)=\# \operatorname{ker}\left(\pi_{\Phi}^{\vee}\right)$. By Corollary 2.4 there is an injection of $\operatorname{ker}\left(\pi_{\Phi}^{\vee}\right)$ into the split torus $T:=\left(\mathcal{B}^{\vee}\right)_{k}^{0} /\left(\mathcal{A}^{\vee}\right)_{k}^{0}$, which has 
dimension $\operatorname{dim}(B)-\operatorname{dim}(A)$. In fact, $\operatorname{since} \operatorname{ker}\left(\pi_{\Phi}^{\vee}\right)$ is constant, it must inject into $T(k) \cong\left(k^{\times}\right)^{\operatorname{dim}(B)-\operatorname{dim}(A)}$. The proposition follows by combining all these facts.

2.3. Formula for the degree. Let $X$ be a proper, smooth, and geometrically connected curve over $K$. Assume $X(K) \neq \emptyset$. Also assume that $X$ has a semistable model over $R$ with a $k$-split degenerate closed fibre. This last condition means that there is a proper flat curve $\mathcal{X}$ over $R$ such that $\mathcal{X}_{K} \cong X$, and all the irreducible components of $\mathcal{X}_{k}$ are isomorphic to $\mathbb{P}_{k}^{1}$ intersecting transversally at $k$ rational points. Let $J=\operatorname{Pic}_{X / K}^{0}$ be the Jacobian variety of $X$, and let $\mathcal{J}$ be the Néron model of $J$ over $R$. From the general theory of Néron models one knows that $J$ has split toric reduction; see [1, Ch.9]. Fix a point $\infty \in X(K)$. Let $E$ be an elliptic curve over $K$. Assume there is a non-trivial morphism $\wp: X \rightarrow E$ which sends $\infty$ to the origin of $E$. Assume further that the homomorphism $\pi: J \rightarrow E$ induced from $\wp$ by the Albanese functoriality realizes $E$ as an optimal quotient of $J$. The morphism $\wp$ can be recovered as the composite of the canonical closed immersion $X \hookrightarrow J$, which sends $\infty$ to the origin, with the quotient map $\pi$. Using the canonical principal polarizations on $J$ and $E$, the homomorphism $E \rightarrow J$ induced from $\wp$ by the Picard functoriality can be identified with $\pi^{\vee}$.

Lemma 2.8. The endomorphism $\pi \circ \pi^{\vee} \in \operatorname{End}_{K}(E)$ is multiplication by $\operatorname{deg}(\wp)$.

Proof. Let more generally $f: X \rightarrow Y$ be a finite morphism of smooth projective geometrically irreducible curves over a field $K$, with $\operatorname{deg} f=n \geq 1$. The map induced on Picard varieties $f^{*}: \mathrm{Pic}_{Y / K} \rightarrow \mathrm{Pic}_{X / K}$ by Picard functoriality is given by $\mathcal{L} \mapsto f^{*} \mathcal{L}$. The map $f_{*}: \mathrm{Pic}_{X / K} \rightarrow \mathrm{Pic}_{Y / K}$ induced by Albanese functoriality is given by $\mathcal{W} \mapsto \operatorname{det}\left(f_{*} \mathcal{W}\right) \otimes_{\mathcal{O}_{Y}} \operatorname{det}\left(f_{*} \mathcal{O}_{X}\right)^{-1}$. We need to show that $f_{*} \circ f^{*}$ : $\operatorname{Pic}_{Y / K} \rightarrow \operatorname{Pic}_{Y / K}$ is just multiplication by $n$. By the Projection Formula, for $\mathcal{L} \in \operatorname{Pic}_{Y / K}$ we have $f_{*}\left(f^{*} \mathcal{L}\right) \cong \mathcal{L} \otimes_{\mathcal{O}_{Y}} f_{*} \mathcal{O}_{X}$. Hence

$$
\begin{gathered}
\operatorname{det}\left(f_{*}\left(f^{*} \mathcal{L}\right)\right) \otimes \mathcal{O}_{Y} \operatorname{det}\left(f_{*} \mathcal{O}_{X}\right)^{-1} \cong \operatorname{det}\left(\mathcal{L} \otimes_{\mathcal{O}_{Y}} f_{*} \mathcal{O}_{X}\right) \otimes_{\mathcal{O}_{Y}} \operatorname{det}\left(f_{*} \mathcal{O}_{X}\right)^{-1} \\
\cong \mathcal{L}^{\otimes n} \otimes_{\mathcal{O}_{Y}} \operatorname{det}\left(f_{*} \mathcal{O}_{X}\right) \otimes_{\mathcal{O}_{Y}} \operatorname{det}\left(f_{*} \mathcal{O}_{X}\right)^{-1} \cong \mathcal{L}^{\otimes n} .
\end{gathered}
$$

Since $E$ is isomorphic to a subvariety of $J$ which has split toric reduction, $E$ has split multiplicative reduction. Its character group $M_{E}$ is isomorphic to $\mathbb{Z}$. Denote by $\rho$ a generator of $M_{E}$ (this is well-defined up to a sign). Let

$$
v_{E}:=\left(\pi^{*}(\rho) \otimes_{\mathbb{Z}} \mathbb{Q}\right) \cap M_{J} \text { and } c:=\#\left(M_{J} / \pi^{*}\left(M_{E}\right)\right)_{\text {tor }} .
$$

In particular, $\pi^{*}(\rho)=c \cdot v_{E}$. Denote the canonical principal polarization on Jacobian varieties by $\theta$.

Proposition 2.9. We have the formula

$$
\# \Phi_{E} \cdot \operatorname{deg} \wp=\# \operatorname{coker}\left(\pi_{\Phi}\right)^{2} \cdot u_{J, \theta}\left(v_{E}, v_{E}\right) \text {. }
$$

Proof. Indeed, according to Theorem 2.5(iv), $\# \Phi_{E}=u_{E, \theta}(\rho, \rho)$. Using bifunctoriality and bilinearity of the monodromy pairing, along with Lemma 2.8, we have

$$
\operatorname{deg} \wp \cdot u_{E, \theta}(\rho, \rho)=u_{E, \theta}\left(\pi_{*} \circ \pi^{*} \rho, \rho\right)=u_{J, \theta}\left(\pi^{*} \rho, \pi^{*} \rho\right)=c^{2} \cdot u_{J, \theta}\left(v_{E}, v_{E}\right) .
$$

On the other hand, we know from (2.4) that $c=\# \operatorname{coker}\left(\pi_{\Phi}: \Phi_{J} \rightarrow \Phi_{E}\right)$. This finishes the proof.

Corollary 2.10. Let $q=\# k$. Then $\operatorname{deg} \wp \leq(q-1) \cdot u_{J, \theta}\left(v_{E}, v_{E}\right)$. 
Proof. Obviously $\# \Phi_{E} \geq \# \operatorname{coker}\left(\pi_{\Phi}\right)$. From Proposition 2.7 we have \# $\operatorname{coker}\left(\pi_{\Phi}\right) \leq$ $(q-1)$. The claim follows from Proposition 2.9.

\section{Proof of the MAIN THEOREM}

Notation. Let $C$ be a smooth, projective, geometrically connected curve over the finite field of $q$ elements $\mathbb{F}_{q}$. Let $F$ be the field of rational functions on $C$. Fix a closed point $\infty$ on $C$ of degree $d_{\infty}$. Denote by $A$ the ring of functions on $C$ which are regular away from $\infty$. Let $K$ be the completion of $F$ at $\infty, R$ be the ring of integers in $K$, and $k$ be the residue field. Let $q_{\infty}:=q^{d_{\infty}}(=\# k)$. Finally, let $G:=\mathrm{GL}(2)$.

3.1. Drinfeld modular curves. The function field analogue of the complex upper half-plane is the Drinfeld upper half-plane

$$
\Omega=\mathbb{P}_{K}^{1}-\mathbb{P}_{K}^{1}(K) .
$$

It has a natural structure of a one-dimensional rigid-analytic space over $K$.

Let $\Lambda$ be a projective $A$-submodule of rank two in $F^{2}$ and

$$
G(\Lambda)=\{\gamma \in G(F) \mid \Lambda \gamma=\Lambda\} .
$$

Fix an ideal $\mathfrak{n}$ of $A$, and let $G(\mathfrak{n})=\operatorname{ker}(G(\Lambda) \rightarrow G(\Lambda / \mathfrak{n} \Lambda))$ be the $\mathfrak{n}$-th congruence subgroup of $G(\Lambda)$. An arithmetic subgroup $\Gamma$ of $G(F)$ is an intermediate group $G(\mathfrak{n}) \subset \Gamma \subset G(\Lambda)$ for suitable $\Lambda$ and $\mathfrak{n}$. The group $\Gamma$ acts on $\Omega$ through linear fractional transformations with finite stabilizers, hence the quotient $\Gamma \backslash \Omega$ exists as a rigid-analytic space over $K$. In fact, it is a theorem of Drinfeld [6] that there is a unique smooth, geometrically irreducible affine algebraic curve $Y_{\Gamma} / K$ such that its analytification $Y_{\Gamma}^{\text {an }}$ is isomorphic to $\Gamma \backslash \Omega$. The curve $Y_{\Gamma}$ is called a Drinfeld modular curve for $\Gamma$. The compactification of $Y_{\Gamma}$ will be denoted by $X_{\Gamma}$. From some general facts about algebraic curves with analytic uniformization it follows that $X_{\Gamma}$ is a Mumford curve, that is, $X_{\Gamma}$ has a flat model over $R$ with $k$-split degenerate closed fibre; see 24. Certain properties of $X_{\Gamma}$ are closely related to the combinatorics of the Bruhat-Tits tree $\mathcal{T}$ of $\mathrm{PGL}_{2}(K)$ and its quotients. There is a pure affinoid covering of $\Omega$ whose associated analytic reduction has an intersection graph isomorphic to $\mathcal{T}$; see $[9, \S \S 1-2]$. We already mentioned that an arithmetic group $\Gamma$ acts on $\Omega$ via fractional linear transformations. This action respects the pure covering of $\Omega$, and hence acts on $\mathcal{T}$. (The group $\Gamma$ also naturally acts on $\mathcal{T}$ as the space of equivalence classes of rank two $R$-submodules of $K^{2}$. One can show that the resulting two actions are canonically isomorphic; see loc.cit.) The quotient graph

$$
\Gamma \backslash \mathcal{T} \cong \mathcal{G} \cup \bigcup h_{i},
$$

is a union of a finite graph $\mathcal{G}$ and a finite number of infinite half-lines $h_{i}$. In $\$ 3.2$ we will see how $\mathcal{G}$ is related to $X_{\Gamma}$.

3.2. Monodromy pairing on $J_{\Gamma}$. Let $J_{\Gamma}$ be the Jacobian variety of $X_{\Gamma}$. As we indicated in $\oint_{2}, J_{\Gamma}$ will have a split toric reduction over $R$. We would like to describe the $\theta$-polarized monodromy pairing $u_{J, \theta}$ on $J_{\Gamma}$ explicitly in terms of the arithmetic group $\Gamma$ and its action on $\Omega$. We state a result from [19, §4] which gives such a description. The details being somewhat lengthy, we simply indicate the main ingredients of the proof. 
First, one needs to construct a semi-stable model of $X_{\Gamma}$ over $\operatorname{Spec}(R)$. It is possible to construct such a model using the rigid-analytic uniformization of $X_{\Gamma}$. More precisely, one needs an explicit admissible affinoid covering of $X_{\Gamma}^{\text {an }}$. This is the subject of a paper by Reversat [22]. Associating a formal affine scheme to each of these affinoids in a standard manner, and then gluing those formal affines, one obtains a proper flat one-dimensional formal scheme over $\operatorname{Spf}(R)$. Grothendieck's algebraization theorem yields a proper flat curve $\mathcal{X}_{\Gamma}$ over $\operatorname{Spec}(R)$ which is a semistable model of $X_{\Gamma}$. We understand $\mathcal{X}_{\Gamma}$ fairly well due to the explicit nature of its construction. For example, the dual graph of the closed fibre $\left(\mathcal{X}_{\Gamma}\right)_{k}$ is isomorphic to the $\mathcal{G}$ appearing in (3.1); see [19, Prop.4.3].

Once we have $\mathcal{X}_{\Gamma}$ it is possible to make the monodromy pairing $u_{J, \theta}$ explicit using the Picard-Lefschetz formula [11, (12.10)]. Consider $\mathcal{G}$ as an oriented graph, that is, keep track of the ordering of the irreducible components through each singular point of $\left(\mathcal{X}_{\Gamma}\right)_{k}$. Let $\alpha$ be an oriented cycle in $\mathcal{G}$. One can describe $\alpha$ by a sequence of oriented edges $\left\{e_{1}, \ldots, e_{n}\right\}$ satisfying $t\left(e_{i}\right)=o\left(e_{i+1}\right)$ for $i=1, \ldots, n-1$ and $t\left(e_{n}\right)=o\left(e_{1}\right)$, where $t(e)$ (resp. $o(e)$ ) denotes the terminus (resp. origin) of the edge $e$. Define a pairing $\phi\left(e_{1}, e_{2}\right)$ between the oriented edges of $\mathcal{G}$ by

$$
\phi\left(e_{1}, e_{2}\right)= \begin{cases}1 & \text { if } e_{1}=e_{2}, \\ -1 & \text { if } e_{1}=\bar{e}_{2}, \\ 0 & \text { otherwise }\end{cases}
$$

(Here the bar denotes the opposite orientation.) For the cycle $\alpha$ put

$$
\phi_{\alpha}(e)=\sum_{i=1}^{n} \phi\left(e, e_{i}\right) .
$$

One easily checks that $\phi_{\alpha}$ depends only on the class of $\alpha$ in $\mathrm{H}_{1}(\mathcal{G}, \mathbb{Z})$, and $\phi_{\alpha+\beta}=$ $\phi_{\alpha}+\phi_{\beta}$. The action of $\Gamma$ on $\Omega$ and on $\mathcal{T}$ factors through its image $\widetilde{\Gamma}$ in $\operatorname{PGL}_{2}(F)$. Also, since $\Gamma$ acts discontinuously, the stabilizer in $\Gamma$ of each edge of $\mathcal{T}$ is finite. Given an edge $e \in \operatorname{Ed}(\mathcal{G})$, denote by $e^{\prime}$ some preimage of $e$ in $\mathcal{T}$, and let $n(e)=$ \# $\operatorname{Stab}_{e^{\prime}} \widetilde{\Gamma}$. It is easy to check that this is well-defined.

Theorem 3.1. Let $M$ be the character group of $\mathcal{J}_{\Gamma}^{0} \times k$, where $\mathcal{J}_{\Gamma}$ is the Néron model of $J_{\Gamma}$ over $R$. Then $M \cong \mathrm{H}_{1}(\mathcal{G}, \mathbb{Z})$, and the $\theta$-polarized monodromy pairing $M \times M \rightarrow \mathbb{Z}$ is given by

$$
u_{J, \theta}(\alpha, \beta)=\sum_{e \in \operatorname{Ed}(\mathcal{G})} n(e) \phi_{\alpha}(e) \phi_{\beta}(e) .
$$

Proof. [19, Cor. 4.4.].

3.3. Harmonic cochains for $\Gamma$. Let $\underline{H}_{!}(\mathcal{T}, \mathbb{C})^{\Gamma}$ be the $\mathbb{C}$-vector space of $\mathbb{C}$-valued cuspidal harmonic cochains for $\Gamma$. This is a group of $\mathbb{C}$-valued currents on the edges of $\mathcal{T}$ which are invariant under the action of $\Gamma$ and have compact support modulo $\Gamma$. For the definition and the properties of this group we refer to [9, §3]. It is known that $\operatorname{dim}_{\mathbb{C}} \underline{H}_{!}(\mathcal{T}, \mathbb{C})^{\Gamma}$ is equal to the genus of $X_{\Gamma}$. There is a natural injective homomorphism loc.cit.

$$
\iota: \mathrm{H}_{1}(\mathcal{G}, \mathbb{Z}) \rightarrow \underline{\mathrm{H}}_{!}(\mathcal{T}, \mathbb{C})^{\Gamma} .
$$

As a consequence of a theorem of Drinfeld [6. Thm.10.3], see also [9, §4], the group $\underline{\mathrm{H}}_{!}(\mathcal{T}, \mathbb{C})^{\Gamma}$ is a subspace of the space of cusp forms on $G\left(\mathbb{A}_{F}\right)$ whose corresponding 
cuspidal representations are special at $\infty$. (Here $\mathbb{A}_{F}$ is the ring of adeles of $F$.) In particular, there is a positive definite pairing on $\underline{H}_{!}(\mathcal{T}, \mathbb{C})^{\Gamma}$ which comes from the Petersson inner product on the cusp forms.

Using the strong approximation theorem, one obtains a push-forward Haar measure on the edges of $\Gamma \backslash \mathcal{T}$ from a Haar measure on $G\left(\mathbb{A}_{F}\right)$, and the Petersson product on $\underline{H}_{!}(\mathcal{T}, \mathbb{C})^{\Gamma}$ with respect to such a measure $\mu$ takes the form, cf. [9, $(4.8)]$,

$$
\left(\varphi_{1}, \varphi_{2}\right)_{\mu}=\sum_{e \in \operatorname{Ed}(\mathcal{G})} \varphi_{1}(e) \varphi_{2}(e) \mu(e)
$$

With notation as in Theorem 3.1 assign a weight to an edge $e$ of the quotient graph

$$
\mu_{\mathrm{gr}}(e)=\frac{1}{2} n(e)^{-1}
$$

It is known that $\mu_{\text {gr }}$ is a push-forward Haar measure on $\Gamma \backslash \mathcal{T}$; see [9, (4.8)]. We have the following crucial fact.

Theorem 3.2. Under the isomorphism of Theorem 3.1, we have

$$
u_{J_{\Gamma}, \theta}(\alpha, \beta)=(\iota(\alpha), \iota(\beta))_{\mu_{\mathrm{gr}}} .
$$

Proof. The homomorphism in (3.2) has a simple description [9, (3.2.6)]. This allows us to give an explicit expression for $(\iota(\alpha), \iota(\beta))_{\mu_{\mathrm{gr}}}$; see the proof of [9, (5.7.1)]. This latter formula matches the formula for $u_{J_{\Gamma}, \theta}(\alpha, \beta)$ in Theorem 3.1 .

3.4. Main theorem. Let $E$ be an elliptic curve over $F$ of conductor $\mathfrak{n}_{E}=\mathfrak{n} \cdot \infty$ and split multiplicative reduction at $\infty$, where $\mathfrak{n}$ is an ideal of $A$. It is known 9] that $E_{/ K}$ is a quotient of $J_{\Gamma}$, where $\Gamma:=\Gamma_{0}(\mathfrak{n})$ is the Hecke congruence subgroup of $G(A)$ of level $\mathfrak{n}$

$$
\Gamma_{0}(\mathfrak{n})=\left\{\left(\begin{array}{ll}
a & b \\
c & d
\end{array}\right) \in G(A) \mid c \in \mathfrak{n}\right\} .
$$

Assume $E_{/ K}$ is an optimal quotient as in Definition 2.6. Let

$$
\wp: X_{\Gamma} \rightarrow E_{/ K}
$$

be the non-trivial morphism obtained by composing the canonical embedding $X_{\Gamma} \hookrightarrow$ $J_{\Gamma}$, which sends the cusp $\infty$ to the origin, with the optimal quotient map.

Let $v_{E} \in \mathrm{H}_{1}(\mathcal{G}, \mathbb{Z})$ be the primitive element (2.5) corresponding to $E$ under the isomorphism $M \cong \mathrm{H}_{1}(\mathcal{G}, \mathbb{Z})$ in Theorem 3.1. There is a natural action of the Hecke algebra on $\mathrm{H}_{1}(\mathcal{G}, \mathbb{Z})$, and this action preserves $\mathbb{Z} v_{E}$. It is known that the map in (3.2) is equivariant under the action of the Hecke algebra; see [9. (9.3.2)]. Hence $\iota\left(v_{E}\right)$ is an eigenform corresponding to $E$. Assume $\iota\left(v_{E}\right)$ is the normalized eigenform; cf. 4.3 Assume also that $E$ is semi-stable.

Theorem 3.3. With previous assumptions and notation,

$$
\operatorname{deg} \wp \leq\left(q_{\infty}-1\right) q^{14 g+5} q^{\operatorname{deg} \mathfrak{n}_{E}}\left(\operatorname{deg} \mathfrak{n}_{E}\right)^{3} .
$$

Proof. By Corollary 2.10 and Theorem 3.2 we have the bound

$$
\operatorname{deg} \wp \leq\left(q_{\infty}-1\right)\left(\iota\left(v_{E}\right), \iota\left(v_{E}\right)\right)_{\mu_{\mathrm{gr}}} .
$$

Let $\pi_{E}$ be the cuspidal automorphic representation corresponding to $E$; cf. 9, (8.2)]. Since we are assuming $\iota\left(v_{E}\right)$ is the normalized newform, Corollary 4.5 gives

$$
\operatorname{deg} \wp \leq\left(q_{\infty}-1\right) q^{2 g-2} q^{\operatorname{deg} \mathfrak{n}_{E}} L\left(1, \operatorname{Sym}^{2} \pi_{E}\right) .
$$


The Langlands correspondence preserves the conductors, so the conductor of $\pi_{E}$ is $\mathfrak{n}_{E}$. Thus, the claim follows from Theorem 4.6.

\section{RANKIN-SELBERG METHOD}

The goal of this section is to bound from above the Petersson norm of a newform in terms of the norm of its conductor. This bound is the required analytic input in the proof of Theorem 3.3. We first apply the Rankin-Selberg method to relate the Petersson norm to the special value of a certain $L$-function, and then use convexity estimates to bound this latter special value. The final result should be well-known to the specialists, but in absence of a convenient reference we will sketch some of the details.

4.1. Conventions. Unless specified otherwise, the following notation is fixed until the end of the paper:

$C$ is a smooth, projective, geometrically irreducible curve over the finite field $\mathbb{F}_{q}$;

$F$ is the field of rational functions on $C$;

$\infty$ is a fixed closed point on $C$;

$A=\mathrm{H}^{0}(C-\infty, \mathcal{O})$ is the ring of regular functions on $C-\{\infty\}$;

$F_{v}$ is the completion of $F$ at the place $v$;

$\varpi_{v}$ is a uniformizer of $F_{v}$;

$\operatorname{ord}_{v}$ is the canonical valuation on $F_{v}$ normalized by $\operatorname{ord}_{v}\left(\varpi_{v}\right)=1$;

$\mathcal{O}_{v}=\left\{x \in F_{v} \mid \operatorname{ord}_{v}(x) \geq 0\right\}$ is the ring of integers in $F_{v}$;

$\mathfrak{p}_{v}=\varpi_{v} \mathcal{O}_{v}$ is the maximal ideal of $\mathcal{O}_{v}$;

$k_{v}$ is the residue field $\mathcal{O}_{v} / \mathfrak{p}_{v}$;

$\operatorname{deg}(v)=\left[k_{v}: \mathbb{F}_{q}\right]$

$q_{v}=q^{\operatorname{deg}(v)}$ is the order of the finite field $k_{v}$;

$\zeta_{v}(s)=\left(1-q_{v}^{-s}\right)^{-1}, s \in \mathbb{C}$, is the zeta function of $F_{v}$;

$\zeta_{F}(s)=\prod_{v} \zeta_{v}(s)$ is the zeta function of $F$;

$\mathbb{A}$ is the ring of adeles of $F$;

$\mathbb{A}^{\times}$is the group of ideles of $F$;

$G=\mathrm{GL}(2)$;

$B$ is the Borel subgroup of upper-triangular matrices of $G$;

$Z$ is the center of $G$;

$\mathcal{K}_{v}=G\left(\mathcal{O}_{v}\right)$ is the standard maximal compact subgroup of $G\left(F_{v}\right)$;

$\mathcal{K}_{0}\left(\mathfrak{n}_{v}\right)=\left\{\left(\begin{array}{ll}a & b \\ c & d\end{array}\right) \in \mathcal{K}_{v} \mid c \in \mathfrak{n}_{v}\right\}$, where $\mathfrak{n}_{v}$ is an ideal of $\mathcal{O}_{v}$, is the Hecke congruence subgroup of $\mathcal{K}_{v}$ of level $\mathfrak{n}_{v}$;

$\mathcal{K}=\prod_{v} G\left(\mathcal{O}_{v}\right)$ is the standard maximal compact subgroup of $G(\mathbb{A})$;

$\mathfrak{n}=\prod_{v} \mathfrak{n}_{v}=\prod_{v} \mathfrak{p}_{v}^{n_{v}}, n_{v} \in \mathbb{Z}$, and almost all $n_{v}$ are equal to 0 , is a Weil divisor on $C$ written multiplicatively;

$\operatorname{deg}(\mathfrak{n})=\sum_{v} n_{v} \operatorname{deg}(v)$

$\mathcal{N}(\mathfrak{n})=q^{\operatorname{deg} \mathfrak{n}}$

$\mathcal{K}_{0}(\mathfrak{n})=\prod_{v} \mathcal{K}_{0}\left(\mathfrak{n}_{v}\right)$, where $\mathfrak{n}$ is a non-negative Weil divisor, is the Hecke congruence subgroup of $\mathcal{K}$ of level $\mathfrak{n}$.

By a quasi-character $\chi$ of a group $H$ we mean a homomorphism $\chi: H \rightarrow \mathbb{C}^{\times}$. A character is a unitary quasi-character. If $H$ is a topological group we will understand that (quasi-)characters are required to be continuous (with $\mathbb{C}^{\times}$given its usual topology). 
We specify Haar measures on several important groups. The local Haar measure $d x_{v}$ of $F_{v}$ will be normalized so that $\int_{\mathcal{O}_{v}} d x_{v}=1$. We will likewise normalize the Haar measure $d^{\times} x_{v}$ on $F_{v}^{\times}$by the condition $\int_{\mathcal{O}_{v}^{\times}} d^{\times} x_{v}=1$; explicitly $d^{\times} x_{v}=$ $\left(1-\frac{1}{q_{v}}\right)^{-1} \frac{d x_{v}}{\left|x_{v}\right|_{v}}$. The measures which we will use on the adeles $\mathbb{A}$ and ideles $\mathbb{A}^{\times}$ will be the restricted product measures $d x=\prod_{v} d x_{v}$ and $d^{\times} x=\prod_{v} d^{\times} x_{v}$.

Now we can normalize a Haar measure on $G\left(F_{v}\right)$. Indeed, $G$ is reductive and hence unimodular, and by the Iwasawa decomposition $G\left(F_{v}\right)=B\left(F_{v}\right) \mathcal{K}_{v}$. Since $\mathcal{K}_{v}$ is compact, and hence unimodular, we normalize the Haar measure $d k_{v}$ to give total volume 1 . On the other hand, $B=\left(Z \times T_{1}\right) \ltimes N$ as algebraic groups. Here $T_{1}$ denotes the subgroup of diagonal matrices whose lower right entry is 1 and $N$ the unipotent upper-triangular group. Since $Z \cong T_{1} \cong \mathbb{G}_{m}$, on $F_{v}$-points we take $d z_{v}$ and $d t_{v}$ to be the transfer of the measure $d^{\times} x_{v}$ from $F_{v}^{\times}$with the previous normalization. On $F_{v}$-points of $N \cong \mathbb{G}_{a}$ we take the transfer measure $d n_{v}$ from $d x_{v}$, again with the previous normalization. Now as one easily checks, cf. [2, p.426], $d_{L} b_{v}=d z_{v} d t_{v} d n_{v}$ is a left Haar measure on $B\left(F_{v}\right)$. Finally on $G\left(F_{v}\right)$ we take the measure $d g_{v}=d k_{v} d_{L} b_{v}$, and on $G(\mathbb{A})$ we take the restricted product measure $\prod_{v} d g_{v}$.

Let $H$ be a locally compact Hausdorff topological group with a right Haar measure $\mu$, and let $\Gamma$ be a discrete subgroup of $H$. The canonical mapping $\varphi: H \rightarrow H / \Gamma$ is a local homeomorphism. It is easily seen that there is a unique measure $\mu^{\prime}$ on $H / \Gamma$ such that, whenever $X$ is a measurable subset of $H$ which is mapped by $\varphi$ bijectively onto its image $X^{\prime}=\varphi(X)$ in $H / \Gamma, \mu^{\prime}\left(X^{\prime}\right)=\mu(X)$. We will call $\mu^{\prime}$ the push-forward measure of $\mu$.

4.2. Eisenstein series. For $s \in \mathbb{C}$, let $\delta_{v}(b, s)$ be the representation of $B\left(F_{v}\right)$ given by its modular quasi-character

$$
\delta_{v}\left(\left(\begin{array}{cc}
y_{1} & x \\
0 & y_{2}
\end{array}\right), s\right)=\left|\frac{y_{1}}{y_{2}}\right|_{v}^{s} .
$$

We consider the induced representation $I_{v}(s)=\operatorname{Ind}_{B\left(F_{v}\right)}^{G\left(F_{v}\right)} \delta_{v}(s)$ consisting of locally constant functions $\Phi_{v}(g, s)$ on $G\left(F_{v}\right)$ such that

$$
\Phi_{v}(b g, s)=\delta_{v}(b, s) \Phi_{v}(g, s)
$$

for $b \in B\left(F_{v}\right)$ and $g \in G\left(F_{v}\right)$. Note that such functions automatically have compact support modulo $B$, and that $Z$ acts trivially in this representation. Moreover, by the Iwasawa decomposition $G\left(F_{v}\right)=B\left(F_{v}\right) \mathcal{K}_{v}$, any $\Phi_{v}(g, s)$ is uniquely determined by its restriction to $\mathcal{K}_{v}$. For an ideal $\mathfrak{n}_{v}$ of $\mathcal{O}_{v}$ we will denote by $\Phi_{\mathfrak{n}_{v}}$ the unique vector whose restriction to $\mathcal{K}_{v}$ is the characteristic function of $\mathcal{K}_{0}\left(\mathfrak{n}_{v}\right) \subseteq \mathcal{K}_{v}$. This is well-defined since $\delta_{v}=1$ on $B\left(F_{v}\right) \cap \mathcal{K}_{v}=B\left(F_{v}\right) \cap \mathcal{K}_{0}\left(\mathfrak{n}_{v}\right)$.

Now we consider the global induced representation $I(s)=\bigotimes_{v} I_{v}(s)$ of $G(\mathbb{A})$, consisting of smooth, right $\mathcal{K}$-finite functions on $G(\mathbb{A})$ which satisfy the global analogue of (4.1). For an entire section $\Phi(g, s) \in I(s)$, we have the Eisenstein series

$$
E(g, s, \Phi)=\sum_{\gamma \in B(F) \backslash G(F)} \Phi(\gamma g, s),
$$

which is absolutely convergent for $\operatorname{Re}(s)>1$. As is well-known, this series has a meromorphic continuation to the whole $s$-plane with a simple pole at $s=1$. Given 
a non-negative divisor $\mathfrak{n}$ on $C$, we will denote by $\Phi_{\mathfrak{n}}(g, s)$ the factorizable section $\bigotimes_{v} \Phi_{\mathfrak{n}_{v}}\left(g_{v}, s\right)$. We allow $\mathfrak{n}$ to be equal to 1 , in which case $\Phi_{\mathfrak{n}}$ restricted to $\mathcal{K}$ is the constant function 1 .

For a finite set $S$ of places of $F$ define

$$
D_{S}(s)=\prod_{v \in S} \zeta_{v}(2 s) / \zeta_{v}(s)
$$

If $S$ is empty we put $D_{S}(s)=1$.

Lemma 4.1. Given a non-negative divisor $\mathfrak{n}$ on $C$, let $S$ be the set of places in the support of $\mathfrak{n}$. Then

$$
\operatorname{Res}_{s=1} E\left(g, s, \Phi_{\mathfrak{n}}\right)=\frac{D_{S}(1)}{\mathcal{N}(\mathfrak{n})} \frac{1}{\zeta_{F}(2)} \operatorname{Res}_{s=1} \zeta_{F}(s) .
$$

Proof. According to Proposition 3.7.5 in [2], any $E(g, s, \Phi)$ has a simple pole at $s=1$ and the residue is independent of $g$. Moreover, as follows from the proof of that proposition, the residue in question is equal to

$$
\operatorname{Res}_{s=1} \int_{\mathbb{A}} \Phi\left(\left(\begin{array}{cc}
0 & -1 \\
1 & 0
\end{array}\right)\left(\begin{array}{ll}
1 & x \\
0 & 1
\end{array}\right), s\right) d x .
$$

We compute this latter quantity for $\Phi_{\mathfrak{n}}$. Since $\Phi_{\mathfrak{n}}$ is factorizable, we have

$$
\int_{\mathbb{A}} \Phi_{\mathfrak{n}}\left(\left(\begin{array}{cc}
0 & -1 \\
1 & 0
\end{array}\right)\left(\begin{array}{ll}
1 & x \\
0 & 1
\end{array}\right), s\right) d x=\prod_{v} \int_{F_{v}} \Phi_{\mathfrak{n}_{v}}\left(\left(\begin{array}{cc}
0 & -1 \\
1 & 0
\end{array}\right)\left(\begin{array}{cc}
1 & x_{v} \\
0 & 1
\end{array}\right), s\right) d x_{v} .
$$

So it is enough to compute the local integrals. First consider a place where $\mathfrak{n}_{v}=1$ (which is the case for almost all $v$ ). When $\operatorname{ord}_{v}\left(x_{v}\right) \geq 0$ the expression in the local integral is equal to 1 by definition. On the other hand, if $\operatorname{ord}_{v}\left(x_{v}\right)<0$, then the integrand is equal to $\left|x_{v}\right|_{v}^{-2 s}$ since

$$
\left(\begin{array}{cc}
0 & -1 \\
1 & 0
\end{array}\right)\left(\begin{array}{cc}
1 & x_{v} \\
0 & 1
\end{array}\right)=\left(\begin{array}{cc}
x_{v}^{-1} & -1 \\
0 & x_{v}
\end{array}\right)\left(\begin{array}{cc}
1 & 0 \\
x_{v}^{-1} & 1
\end{array}\right)
$$

The volume of $\mathfrak{p}_{v}^{-m}-\mathfrak{p}_{v}^{-(m-1)}, m>1$, is equal to $q_{v}^{m}\left(1-q_{v}^{-1}\right)$, as we normalized the local Haar measure by $\int_{\mathcal{O}_{v}} d x_{v}=1$. Finally,

$$
\begin{aligned}
\int_{F_{v}} \Phi_{\mathfrak{n}_{v}}\left(\left(\begin{array}{cc}
0 & -1 \\
1 & 0
\end{array}\right)\left(\begin{array}{cc}
1 & x_{v} \\
0 & 1
\end{array}\right), s\right) d x_{v} & =1+\sum_{m=1}^{\infty} \frac{1}{q_{v}^{2 m s}} q_{v}^{m}\left(1-q_{v}^{-1}\right) \\
& =\frac{\zeta_{v}(2 s-1)}{\zeta_{v}(2 s)} .
\end{aligned}
$$

A similar calculation for the case $\operatorname{ord}_{v}\left(\mathfrak{n}_{v}\right)>0$ gives

$$
\int_{F_{v}} \Phi_{\mathfrak{n}_{v}}\left(\left(\begin{array}{cc}
0 & -1 \\
1 & 0
\end{array}\right)\left(\begin{array}{cc}
1 & x_{v} \\
0 & 1
\end{array}\right), s\right) d x_{v}=\left(1-q_{v}^{-1}\right) \frac{\zeta_{v}(2 s-1)}{q_{v}^{\operatorname{ord}_{v}\left(\mathfrak{n}_{v}\right)(2 s-1)}}
$$

From these two calculations the claim of the lemma easily follows.

It is perhaps instructive to describe the Eisenstein series $E(g, s, \Phi)$ more explicitly as functions on Bruhat-Tits trees and to compare them with the series considered in [8] and [18]. We give one example. 
Example 4.2. Let $A=\mathbb{F}_{q}[t]$ be the ring of polynomials with coefficients in $\mathbb{F}_{q}$, $F=\mathbb{F}_{q}(t)$ be the fraction field of $A$, and $\infty=1 / t$ be the place of $F$ associated to the degree of polynomials as a valuation on $A$. It is clear that $F$ is the function field of $\mathbb{P}_{\mathbb{F}_{q}}^{1}$. Let $\mathfrak{n}$ be a non-negative divisor with support in $\operatorname{Spec}(A)$. Denote by $\mathcal{K}_{f}$ the restricted product $\prod_{v \neq \infty} \mathcal{K}_{v} ;$ similarly, write $\mathcal{K}_{0, f}(\mathfrak{n})=\prod_{v \neq \infty} \mathcal{K}_{0}\left(\mathfrak{n}_{v}\right)$. Let $\Gamma=$ $G(A)=G(F) \cap \mathcal{K}_{f}$, and $\Gamma_{0}(\mathfrak{n})=\left\{\left(\begin{array}{ll}a & b \\ c & d\end{array}\right) \in \Gamma \mid c \equiv 0 \bmod \mathfrak{n}\right\}=G(F) \cap \mathcal{K}_{0, f}(\mathfrak{n})$. As det $: \mathcal{K}_{0, f}(\mathfrak{n}) \rightarrow \mathcal{O}_{f}^{\times}$is surjective, by the strong approximation theorem,

$$
G(\mathbb{A})=G(F) G\left(F_{\infty}\right) \mathcal{K}_{0, f}(\mathfrak{n}) .
$$

Since $E\left(g, s, \Phi_{\mathfrak{n}}\right)$ is left $G(F)$ and right $\mathcal{K}_{0}(\mathfrak{n})$ invariant, it suffices to evaluate it for $g \in G\left(F_{\infty}\right)$, which we view as an element of $G(\mathbb{A})$ with trivial components at all places except for $\infty$. Observe that $B(F) \backslash G(F) \cong \Delta \backslash \Gamma$, where $\Delta=\left\{\left(\begin{array}{ll}a & b \\ 0 & d\end{array}\right) \in \Gamma\right\}$. Let $\gamma \in \Gamma$. Then $\Phi_{\mathfrak{n}}(\gamma g, s)=\left(\prod_{v \neq \infty} \Phi_{\mathfrak{n}_{v}}(\gamma, s)\right) \cdot \Phi_{\infty}(\gamma g, s)$ is equal to $\Phi_{\infty}(\gamma g, s)$ or 0 depending on whether $\gamma \in \Gamma_{0}(\mathfrak{n})$ or not. So

$$
E\left(g, s, \Phi_{\mathfrak{n}}\right)=\sum_{\gamma \in \Delta \backslash \Gamma_{0}(\mathfrak{n})} \Phi_{\infty}(\gamma g, s) .
$$

It remains to make $\Phi_{\infty}$ explicit. Since $\Phi_{\infty}$ is invariant under the right action of $Z\left(F_{\infty}\right) \mathcal{K}_{\infty}$, we may regard it as a function on

$$
G\left(F_{\infty}\right) / Z\left(F_{\infty}\right) \mathcal{K}_{\infty} \cong B\left(F_{\infty}\right) /\left(B\left(F_{\infty}\right) \cap \mathcal{K}_{\infty}\right) Z\left(F_{\infty}\right) .
$$

We easily see that each matrix in this set is represented by a unique matrix of the form $\left(\begin{array}{cc}\varpi_{\infty}^{k} & u \\ 0 & 1\end{array}\right)$, where $k \in \mathbb{Z}$, and $u \in F_{\infty} \bmod \mathfrak{p}_{\infty}^{k}$. By definition

$$
\Phi_{\infty}\left(\left(\begin{array}{cc}
\varpi_{\infty}^{k} & u \\
0 & 1
\end{array}\right), s\right)=q^{-k s} .
$$

From this point of view $E\left(g, s, \Phi_{\mathfrak{n}}\right)$ is a function on the vertices of the Bruhat-Tits tree of $\mathrm{PGL}_{2}\left(F_{\infty}\right)$, an infinite tree in which every vertex is adjacent to exactly $q+1$ other vertices.

4.3. Rankin-Selberg integral. Let $(\pi, V)$ be an infinite-dimensional irreducible cuspidal representation of $G(\mathbb{A})$ with central character $\omega$. Define the conductor $\mathfrak{n}_{\pi}$ of $\pi$ as in [3]; see also [10, Thm. 4.24]. Since $\pi$ is almost everywhere unramified, $\mathfrak{n}_{\pi}$ is a non-negative Weil devisor on $C$. The local $L$-functions of $\pi$ are well-understood; cf. [2, §4.7]. If $\left(\pi_{v}, V_{v}\right)=\pi\left(\chi_{1}, \chi_{2}\right)$ is an irreducible principal series representation, then

$$
L\left(s, \pi_{v}\right)=\left(1-\alpha_{1} q_{v}^{-s}\right)^{-1}\left(1-\alpha_{2} q_{v}^{-s}\right)^{-1},
$$

where $\alpha_{i}=\chi_{i}\left(\varpi_{v}\right)$ if $\chi_{i}$ is unramified and $\alpha_{i}=0$ otherwise. If $\left(\pi_{v}, V_{v}\right)=\sigma\left(\chi_{1}, \chi_{2}\right)$, where $\left(\chi_{1} \chi_{2}^{-1}\right)(y)=|y|_{v}^{-1}$, is a special representation, then

$$
L\left(s, \pi_{v}\right)=\left(1-\alpha_{2} q_{v}^{-s}\right)^{-1},
$$

with $\alpha_{2}$ as above. Finally, if $\pi_{v}$ is supercuspidal, then $L\left(s, \pi_{v}\right)=1$.

If $\pi_{v} \cong \pi\left(\chi_{1}, \chi_{2}\right)$ is a principal series representation of $G\left(F_{v}\right)$ with unramified quasi-characters $\chi_{i}$ on $F_{v}^{\times}$, then by the Ramanujan conjecture, proved for GL(2) over function fields by Drinfeld, $\alpha_{1, v}$ and $\alpha_{2, v}$ are complex conjugates of each other with absolute value 1 . 
Write $L\left(s, \pi_{v}\right)=\left(1-\alpha_{1, v} q_{v}^{-s}\right)^{-1}\left(1-\alpha_{2, v} q_{v}^{-s}\right)^{-1}$ uniformly for all types of local representations $\pi_{v}$ (so $\alpha_{1, v}=0$ if $\pi_{v}$ is special and $\alpha_{1, v}=\alpha_{2, v}=0$ if it is supercuspidal). Define

$$
L\left(s, \operatorname{Sym}^{2} \pi_{v}\right):=\left(1-\alpha_{1, v}^{2} q_{v}^{-s}\right)^{-1}\left(1-\alpha_{1, v} \alpha_{2, v} q_{v}^{-s}\right)^{-1}\left(1-\alpha_{2, v}^{2} q_{v}^{-s}\right)^{-1}
$$

and

$$
L\left(s, \operatorname{Sym}^{2} \pi\right):=\prod_{v} L\left(s, \operatorname{Sym}^{2} \pi_{v}\right) .
$$

Let $\phi \in V$ be a newform attached to $\pi$. The automorphic form $\phi$ is unique up to a scalar [3, and we will always assume that it is normalized by requiring that the local Whittaker functions $W_{\phi, v}(1)=1$ at all places $v$ (we implicitly fix a non-trivial additive character $\psi$ of the compact group $\mathbb{A} / F)$. Such a $\phi$ is well-defined. Let $\bar{\phi}$ be the complex conjugate of the newform $\phi$. The product $\phi(g) \bar{\phi}(g) E\left(g, s, \Phi_{\mathfrak{n}_{\pi}}\right)$ is invariant under $Z(\mathbb{A}) G(F)$ acting on the left and under $\mathcal{K}_{0}\left(\mathfrak{n}_{\pi}\right)$ acting on the right.

Assume that the conductor $\mathfrak{n}_{\pi}$ is square-free, and let $S$ be the set of places in the support of $\mathfrak{n}_{\pi}$ (that is, the set of places where $\pi$ is ramified). The basic formula, which follows from the Rankin-Selberg unfolding method, is

$$
\int_{\mathbf{Y}\left(\mathfrak{n}_{\pi}\right)} \phi(g) \bar{\phi}(g) E_{\mathfrak{n}_{\pi}}(g, s) d g=q^{(g-1) s} D_{S}(s) \frac{\zeta_{F}(s)}{\zeta_{F}(2 s)} L\left(s, \operatorname{Sym}^{2} \pi\right),
$$

where $\mathbf{Y}\left(\mathfrak{n}_{\pi}\right):=Z(\mathbb{A}) G(F) \backslash G(\mathbb{A}) / \mathcal{K}_{0}\left(\mathfrak{n}_{\pi}\right)$ and $g$ on the right hand-side is the genus of $C$. This relation can be proved by essentially repeating the calculation in [2, §3.8] and taking special care of the ramified places. We omit the details of these calculations. Denote

$$
(\phi, \phi):=\int_{\mathbf{Y}\left(\mathfrak{n}_{\pi}\right)} \phi(g) \bar{\phi}(g) d g .
$$

Taking the residues at $s=1$ on both sides of (4.3) and using Lemma 4.1, we get

$$
(\phi, \phi)=q^{g-1} \mathcal{N}\left(\mathfrak{n}_{\pi}\right) L\left(1, \operatorname{Sym}^{2} \pi\right) .
$$

Remark 4.3. The assumption on $\mathfrak{n}_{\pi}$ being square-free is not crucial for computing the Rankin-Selberg integral (although it does simplify the calculation). We make this assumption mostly for $L\left(s, \mathrm{Sym}^{2} \pi\right)$ to have a functional equation; this is used in 4.4 . If we let $\rho$ be the 2 -dimensional $\ell$-adic representation of $\operatorname{Gal}\left(F^{\text {sep }} / F\right)$ associated to $\pi$ via Langlands correspondence, then, in notations of $\$ 4.4, L\left(s, \mathrm{Sym}^{2} \pi_{v}\right)=$ $L_{v}\left(\operatorname{Sym}^{2} \rho, s\right)$ if and only if $\left(\operatorname{Sym}^{2} V_{\ell}\right)^{I_{v}}=\operatorname{Sym}^{2}\left(V_{\ell}^{I_{v}}\right)$ for all $v$, where $I_{v}$ is the inertia group at $v$. This happens, for example, if the conductor is square-free. Even without assuming $\mathfrak{n}_{\pi}$ is square-free, it is possible to deduce a bound on $\left|L\left(1, \operatorname{Sym}^{2} \pi\right)\right|$ (which is what we are interested in) from a bound on $\left|L\left(\operatorname{Sym}^{2} \rho, 1\right)\right|$ by studying the local factors $L_{v}\left(\operatorname{Sym}^{2} \rho, s\right)$ at the places where $\operatorname{ord}_{v}\left(\mathfrak{n}_{\pi}\right) \geq 2$. Hence the assumption on square-freeness can be completely avoided at the expense of making the argument somewhat longer.

Let $\mathbf{W}_{\mathrm{sp}}(\mathfrak{n})$ be the space of automorphic cusp forms whose corresponding representations have conductor $\mathfrak{n} \cdot \infty$, for some ideal $\mathfrak{n}$ in $A$, and are special at $\infty$. This space is finite dimensional. Let $\mathcal{K}_{0, f}(\mathfrak{n})=\prod_{v \neq \infty} \mathcal{K}_{0}\left(\mathfrak{n}_{v}\right)$, and let $\mathbb{A}_{f}$ be the finite adeles, i.e., $\mathbb{A}=\mathbb{A}_{f} \cdot F_{\infty}$. The strong approximation theorem implies that the determinant map induces a bijection

$$
G(F) \backslash G\left(\mathbb{A}_{f}\right) / \mathcal{K}_{f, 0}(\mathfrak{n}) \stackrel{\sim}{\longrightarrow} \operatorname{Pic}(A) .
$$


Choose a system of representatives $\aleph$ for this finite set. Let

$$
\Gamma_{x}:=G(F) \cap x \mathcal{K}_{0, f}(\mathfrak{n}) x^{-1}
$$

be the intersection in $G\left(\mathbb{A}_{f}\right)$. It is not hard to show, cf. [9, (4.5.4)], that

$$
\begin{aligned}
Z\left(F_{\infty}\right) G(F) \backslash G(\mathbb{A}) / \mathcal{K}_{0}(\mathfrak{n} \cdot \infty) & \cong \coprod_{x \in \aleph} Z\left(F_{\infty}\right) \Gamma_{x} \backslash G\left(F_{\infty}\right) / \mathcal{K}_{0}\left(\mathfrak{p}_{\infty}\right) \\
& =\coprod_{x \in \aleph} \operatorname{Ed}\left(\Gamma_{x} \backslash \mathcal{T}\right) .
\end{aligned}
$$

One can think of $\mathbf{W}_{\mathrm{sp}}(\mathfrak{n})$ as the space of $\mathbb{C}$-valued functions on the above double coset set which satisfy a cuspidality condition; cf. [9. (4.4)]. The following key fact is due to Drinfeld [9, (4.7.6)]

$$
\mathbf{W}_{\mathrm{sp}}(\mathfrak{n}) \cong \bigoplus_{x \in \aleph} \underline{\mathrm{H}}_{!}(\mathcal{T}, \mathbb{C})^{\Gamma_{x}}
$$

Now let $E$ be an elliptic curve over $F$ of conductor $\mathfrak{n}_{E}:=\mathfrak{n} \cdot \infty$. Assume the reduction of $E$ at $\infty$ is split multiplicative. Let $\pi_{E}$ be the cuspidal representation corresponding to $E$. The conductor of $\pi_{E}$ is $\mathfrak{n}_{E}$, and $\left(\pi_{E}\right)_{\infty}$ is the special representation. Let $\phi_{E}$ be the (normalized) newform attached to $\pi_{E}$. Then $\phi_{E} \in \mathbf{W}_{\mathrm{sp}}(\mathfrak{n})$ and is an eigenform for the Hecke algebra. It is conventional to normalize the Langlands correspondence in such a way that $\pi_{E}$ has trivial central character, so $\phi_{E}$ is invariant under the action of $Z(\mathbb{A})$; see [9, (8.2.4)]. Using (4.6), we can write $\phi_{E}=\sum_{x \in \mathcal{N}} \phi_{x}$ with $\phi_{x} \in \underline{\mathrm{H}}_{!}(\mathcal{T}, \mathbb{C})^{\Gamma_{x}}$.

We have fixed a Haar measure on $G(\mathbb{A})$ in $\$ 4.1$ which we denote by $\mu_{\text {can }}$. This measure induces a certain push-forward measure on each $\Gamma_{x} \backslash \mathcal{T}$ which we again denote by $\mu_{\text {can }}$. On the other hand, in $₫ 3.3$ we explicitly described a measure on the edges of the quotient graph, which we denoted by $\mu_{\mathrm{gr}}$. Since the Haar measure on $G(\mathbb{A})$ is unique up to a constant multiple, $\mu_{\text {can }}$ and $\mu_{\mathrm{gr}}$ differ by a fixed constant. It is easy to see that the Petersson norms $\left(\phi_{x}, \phi_{x}\right)_{\mu}, x \in \aleph$, are all equal to each other (with respect to either measure). Moreover, we have the following equality.

Proposition 4.4. Fix some $x \in \aleph$. Then

$$
\left(\phi_{E}, \phi_{E}\right)=q^{1-g}\left(\phi_{x}, \phi_{x}\right)_{\mu_{\mathrm{gr}}},
$$

where $g$ is the genus of the base curve $C$.

Proof. From what was said, we deduce that $\left(\phi_{E}, \phi_{E}\right)$ and $\left(\phi_{x}, \phi_{x}\right)_{\mu_{\mathrm{gr}}}$ differ by a non-zero constant which depends possibly on $C$ and $\infty$ (but is independent of $\phi$ ). To get this constant it is enough to compare the volume of $\mathbf{Y}(\mathfrak{n} \cdot \infty)$ with respect to $\mu_{\text {can }}$ to the volume of $\operatorname{Ed}\left(\Gamma_{x} \backslash \mathcal{T}\right)$ with respect to $\mu_{\mathrm{gr}}$ for some fixed $x \in \aleph$. For simplicity we fix $x=1$ and omit it from notation. First we assume $\Gamma=G(A)$, i.e., $\mathfrak{n}=1$. From [23, $\S I I .2 .3]$ we have

$$
\operatorname{Vol}_{\mathrm{gr}}(\Gamma \backslash \mathcal{T})=\left(q_{\infty}+1\right) \zeta_{F}(-1)
$$

Let $M>1$ be a fixed integer. Define a function on $B(\mathbb{A})$ by

$$
\delta_{M}\left(\left(\begin{array}{cc}
y_{1} & x \\
0 & y_{2}
\end{array}\right), s\right)= \begin{cases}\left|\frac{y_{1}}{y_{2}}\right|^{s} & \text { if }\left|\frac{y_{1}}{y_{2}}\right| \leq M \\
0 & \text { otherwise }\end{cases}
$$


Let $\Phi_{\infty}^{M}(g, s)$ be the factorizable locally constant function on $G(\mathbb{A})$ whose restriction to $\mathcal{K}$ is the characteristic function of $\mathcal{K}_{0}(\infty)$, and which satisfies

$$
\Phi_{\infty}^{M}(b g, s)=\delta_{M}(b, s) \Phi_{\infty}^{M}(g, s)
$$

for $g \in G(\mathbb{A})$ and $b \in B(\mathbb{A})$. One easily checks that this is well-defined. Define a truncated Eisenstein series

$$
E_{\infty}^{M}(g, s)=\sum_{\gamma \in B(F) \backslash G(F)} \Phi_{\infty}^{M}(\gamma g, s) .
$$

We modify the Eisenstein series in this manner to make the integrals below convergent. The Rankin-Selberg method [2, §3.8] yields

$$
\int_{\mathbf{Y}(\infty)} E_{\infty}^{M}(g, s) d g=\operatorname{Vol}_{\mathrm{can}}(\mathbb{A} / F) \operatorname{Vol}_{\mathrm{can}}\left(\mathbb{A}^{\times} / F^{\times}\right) \frac{M^{s-1}}{1-q^{1-s}} .
$$

Since $\operatorname{Res}_{s=1} E_{\infty}^{M}(g, s)=\operatorname{Res}_{s=1} E\left(g, s, \Phi_{\infty}\right)$, taking the residues on both sides of this expression and using Lemma 4.1, we get

$$
\frac{\operatorname{Res}_{s=1} \zeta_{F}(s)}{\left(1+q_{\infty}\right) \zeta_{F}(2)} \cdot \operatorname{Vol}_{\text {can }}(\mathbf{Y}(\infty))=\operatorname{Vol}_{\text {can }}(\mathbb{A} / F) \operatorname{Vol}_{\text {can }}\left(\mathbb{A}^{\times} / F^{\times}\right) \frac{1}{\log (q)}
$$

On the other hand, as is well-known 25.

$$
\operatorname{Vol}_{\text {can }}(\mathbb{A} / F)=q^{g-1} \text { and } \operatorname{Vol}_{\text {can }}\left(\mathbb{A}^{\times} / F^{\times}\right)=\frac{\# \operatorname{Pic}_{C / \mathbb{F}_{q}}^{0}\left(\mathbb{F}_{q}\right)}{q-1} .
$$

Since $\# \operatorname{Pic}_{C / \mathbb{F}_{q}}^{0}\left(\mathbb{F}_{q}\right) /(q-1)=\log (q) \cdot \operatorname{Res}_{s=0} \zeta_{F}(s)$ and the functional equation of $\zeta_{F}(s)$ is $\zeta_{F}(1-s)=\left(q^{1-2 s}\right)^{1-g} \zeta_{F}(s)$, we get $\operatorname{Vol}_{\text {can }}(\mathbf{Y}(\infty))=q^{1-g}\left(q_{\infty}+1\right) \zeta_{F}(-1)$. This proves the proposition for $\mathfrak{n}=1$.

For an arbitrary $\mathfrak{n}$, it is not hard to check that

$$
\operatorname{Vol}_{\text {can }}(\mathbf{Y}(\mathfrak{n} \cdot \infty))=\left[\mathcal{K}_{0}(\infty): \mathcal{K}_{0}(\mathfrak{n} \cdot \infty)\right] \operatorname{Vol}_{\text {can }}(\mathbf{Y}(\infty))
$$

and

$$
\operatorname{Vol}_{\text {gr }}\left(\Gamma_{0}(\mathfrak{n}) \backslash \mathcal{T}\right)=\left[\Gamma: \Gamma_{0}(\mathfrak{n})\right] \operatorname{Vol}_{\text {gr }}(\Gamma \backslash \mathcal{T}) .
$$

Since $\left[\mathcal{K}_{0}(\infty): \mathcal{K}_{0}(\mathfrak{n} \cdot \infty)\right]=\left[\Gamma: \Gamma_{0}(\mathfrak{n})\right]$, the proposition follows in general.

Let $\iota\left(v_{E}\right)$ be as in 3.4 . Our assumption is $\iota\left(v_{E}\right)=\phi_{x}$ with $x=1$.

Corollary 4.5. There is the equality

$$
\left(\iota\left(v_{E}\right), \iota\left(v_{E}\right)\right)_{\mu_{\mathrm{gr}}}=q^{2 g-2} \mathcal{N}\left(\mathfrak{n}_{E}\right) L\left(1, \mathrm{Sym}^{2} \pi_{E}\right) .
$$

Proof. Follows from (4.4) and Proposition 4.4.

4.4. Bounds on special values of $L$-functions. Let $\pi$ be a cuspidal representation of $G(\mathbb{A})$. Assume $\widehat{\pi} \cong \pi$, where $\widehat{\pi}$ is the contragredient of $\pi$. (This is the case, for example, when $\pi$ has a trivial central character.) In this subsection we give an upper bound on $\left|L\left(1, \mathrm{Sym}^{2} \pi\right)\right|$ in terms of the degree of the conductor, which finishes the analytic calculations required in the proof of the main theorem, Theorem 3.3 . 
Theorem 4.6. Let $g$ be the genus of the base curve $C$, let $\mathfrak{n}$ be the conductor of $\pi$, and let $n \geq 1$. If $\mathfrak{n}$ is square-free and $\operatorname{deg} \mathfrak{n}>0$, then

$$
\left|L\left(s, \operatorname{Sym}^{n} \pi\right)\right| \leq q^{(4 g+2)(n+1)+\frac{n}{2}} \mathcal{N}(\mathfrak{n})^{\frac{n}{2}(1-\operatorname{Re}(s))}(\operatorname{deg} \mathfrak{n})^{n+1}
$$

for $0 \leq \operatorname{Re}(s) \leq 1$. In particular,

$$
\left|L\left(1, \operatorname{Sym}^{n} \pi\right)\right| \leq q^{(4 g+2)(n+1)+\frac{n}{2}}(\operatorname{deg} \mathfrak{n})^{n+1} .
$$

Proof. We only indicate the main steps which go into the proof of this theorem and refer to [17, Thm. A.4] for the details.

It seems to be more convenient here to work with Galois representations rather than with cuspidal representations. Since Lafforgue proved the Langlands correspondence for $\operatorname{GL}(n)$ over function fields, this will be an equivalent problem; see also Remark 4.3. Choose a separable closure $F^{\text {sep }}$ of $F$ and let $\mathcal{G}=\operatorname{Gal}\left(F^{\mathrm{sep}} / F\right)$ be the absolute Galois group of $F$. Fix a prime $\ell$ not equal to the characteristic of $F$. Let $\rho: \mathcal{G} \rightarrow \operatorname{GL}\left(V_{\ell}\right)$ be an irreducible $\ell$-adic representation of $\mathcal{G}$, where $V_{\ell}$ is a 2-dimensional vector space over some extension of $\mathbb{Q}_{\ell}$. Assume $\rho$ is self-dual and the determinant quasi-character $\operatorname{det}(\rho)$ has finite order. Denote by $\operatorname{Sym}^{n} \rho$ the irreducible $(n+1)$-dimensional $\ell$-adic representation of $\mathcal{G}$ obtained from the action of $\mathcal{G}$ on the symmetric tensors of $V_{\ell}^{\otimes n}$ via $\rho$. Let $L\left(\operatorname{Sym}^{n} \rho, s\right)$ be Grothendieck's $L$-function for $\operatorname{Sym}^{n} \rho$.

Drinfeld's proof of the Langlands conjecture for GL(2) over function fields implies that $\rho$ is pure of weight 0 , i.e., for any place $v$ where $\rho$ is unramified, the images of the geometric Frobenius eigenvalues $\alpha_{1, v}$ and $\alpha_{2, v}$ under any embedding of $\overline{\mathbb{Q}}_{\ell}$ into $\mathbb{C}$ are of absolute value 1 . Thus, for $\operatorname{Re}(s)=1+\epsilon$, with $\epsilon>0$,

$$
\left|L_{v}\left(\operatorname{Sym}^{n} \rho, s\right)\right| \leq \zeta_{F_{v}}(1+\epsilon)^{n+1} .
$$

The same estimate is also valid at the ramified places by [5, Lem. 1.8.1] (in fact the estimates at the ramified places are even somewhat better). Putting the local factors together, we get $\left|L\left(\operatorname{Sym}^{n} \rho, s\right)\right| \leq \zeta_{F}(1+\epsilon)^{n+1}$ for $\operatorname{Re}(s)=1+\epsilon$. From Grothendieck's theory of $L$-functions of $\ell$-adic representations, $L\left(\operatorname{Sym}^{n} \rho, s\right)$ is an entire function [4, §10]; in fact it is a polynomial in $q^{-s}$, since we assumed $\rho$ is irreducible. Moreover, as we also assumed $\rho$ to be self-dual, Grothendieck's functional equation for $L\left(\operatorname{Sym}^{n} \rho, s\right)$ takes the form

$$
L\left(\operatorname{Sym}^{n} \rho, s\right)=\varepsilon\left(\operatorname{Sym}^{n} \rho, s\right) L\left(\operatorname{Sym}^{n} \rho, 1-s\right),
$$

where $\varepsilon\left(\operatorname{Sym}^{n} \rho, s\right)$ is a monomial in $\mathbb{C}\left[q^{-s}\right]$. By restricting $\rho$ to each local Weil group $W_{F_{v}} \hookrightarrow \operatorname{Gal}\left(F^{\mathrm{sep}} / F\right)$, we obtain an $\ell$-adic representation $\rho_{v}$ of $W_{F_{v}}$. Next, one carefully studies the $\varepsilon$-factor of $\operatorname{Sym}^{n} \rho_{v}$ and obtains a lower bound on its absolute value in terms of the degree of the conductor $\mathfrak{n}_{v}$ of $\rho_{v}$. Here the formulae in [4, $\S \S 4-5]$ are indispensable. These estimates imply a bound on the absolute value of the global $\varepsilon$-factor in terms of $\operatorname{deg} \mathfrak{n}_{\rho}$, as by a theorem of Laumon [12, Thm. 0.4] the global $\varepsilon$-factor can be decomposed as a product of local factors. From this bound and the functional equation we get an explicit upper bound on $\left|L\left(\operatorname{Sym}^{n} \rho, 1-s\right)\right|$ for $\operatorname{Re}(s)=1+\epsilon$. Since $L\left(\operatorname{Sym}^{n} \rho, s\right)$ is entire and bounded in the vertical strips one can apply Rademacher's version of the Phragmén-Lindelöf theorem, [20, Thm.2], to conclude that in the vertical strip $-\epsilon \leq \operatorname{Re}(s) \leq 1+\epsilon$

$$
\left|L\left(\operatorname{Sym}^{n} \rho, s\right)\right| \leq\left(q^{(g-1)(n+1)} \mathcal{N}\left(\mathfrak{n}_{\rho}\right)^{\frac{n}{2}}\right)^{(1+\epsilon-\operatorname{Re}(s))} \zeta_{F}(1+\epsilon)^{n+1} .
$$


Recall that by Weil's theorem

$$
\zeta_{F}(s)=\frac{P(s)}{\left(1-q^{-s}\right)\left(1-q \cdot q^{-s}\right)},
$$

where $P(s)=\prod_{i=1}^{2 g}\left(1-a_{i} q^{-s}\right)$, with $\left|a_{i}\right|=\sqrt{q}$. Now it is easy to deduce the required bounds for $L\left(\operatorname{Sym}^{n} \rho, s\right)$ by choosing $\epsilon=\left(\operatorname{deg} \mathfrak{n}_{\rho}\right)^{-1}$. If we let $\rho$ be the $\ell$-adic representation attached to $\pi$ via Langlands correspondence, then the claim follows for $\pi$ too as $\mathfrak{n}_{\rho}=\mathfrak{n}_{\pi}$.

\section{ACKNOWLEDGEMEnTS}

This paper was completed while I was visiting the Max-Planck-Institut für Mathematik in Bonn. I thank the staff and the members of the institute for their hospitality. I also thank C. Skinner, K. Soundararajan, and especially B. Conrad for helpful discussions related to the subject of the paper.

\section{REFERENCES}

[1] S. Bosch, W. Lütkebohmert, and M. Raynaud, Néron models, Springer, 1990. MR 1045822 (91i:14034)

[2] D. Bump, Automorphic forms and representations, Cambridge Univ. Press, 1998. MR.1431508 (97k:11080)

[3] W. Casselman, On some results of Atkin and Lehner, Math. Ann. 201 (1973), 301-314. MR0337789 (49:2558)

[4] P. Deligne, Les constantes des équations fonctionnelles des fonctions L, in Modular functions of one variable II, LNM 349, Springer (1973), 501-598. MR0349635 (50:2128)

[5] P. Deligne, La conjecture de Weil. II, Publ. Math. IHES 52 (1980), 137-252. MR0601520 (83c:14017)

[6] V. Drinfeld, Elliptic modules, Math. Sbornik 94 (1974), 594-627. MR0384707 (52:5580)

[7] E.-U. Gekeler, Analytic construction of Weil curves over function fields, J. Théor. Nombres Bordeaux 7 (1995), 27-49. MR1413565 (97g:11060)

[8] E.-U. Gekeler, Improper Eisenstein series on Bruhat-Tits trees, manuscripta math. 86 (1995), 367-391. MR.1323798 (95m:11043)

[9] E.-U. Gekeler and M. Reversat, Jacobians of Drinfeld modular curves, J. Reine Angew. Math. 476 (1996), 27-93. MR.1401696 (97f:11043)

[10] S. Gelbart, Automorphic forms on adele groups, Princeton Univ. Press, 1975. MR0379375 $(52: 280)$

[11] A. Grothendieck, Modèles de Néron et monodromie, SGA 7, Exposé IX, 1972.

[12] G. Laumon, Les constantes des équations fonctionnelles des fonctions $L$ sur un corps global de caractéristique positive, C. R. Acad. Sci. Paris Sér. I Math. 298 (1984), 181-184. MR0741090 (85j:11170)

[13] L. Mai and M. Ram Murty, The Phragmén-Lindelöf theorem and modular elliptic curves, Contemporary Math. 166 (1994), 335-340. MR.1284072 (95g:11049)

[14] J. S. Milne, Arithmetic duality theorems, Academic Press, 1986. MR0881804 (88e:14028)

[15] M. Ram Murty, Bounds for congruence primes, Proc. Sympos. Pure Math. 66 (1999), 177192. MR:1703750 (2000g:11038)

[16] F. Oort and J. Tate, Group schems of prime order, Ann. scient. Éc. Norm. Sup. 3 (1970), 1-21. MR0265368 (42:278)

[17] M. Papikian, Pesenti-Szpiro inequality for optimal elliptic curves, J. Number Theory 114 (2005), 361-393. MR2167975 (2006f:11062)

[18] M. Papikian, On the degree of modular parametrizations over function fields, J. Number Theory 97 (2002), 317-349. MR1942964 (2004c:11104)

[19] M. Papikian, On component groups of Jacobians of Drinfeld modular curves, Ann. Inst. Fourier 54 (2004), 2163-2199. MR 2139692 (2006b:11060)

[20] H. Rademacher, On the Phragmén-Lindelöf theorem and some applications, Math. Z. 72 (1959), 192-204. MR0117200 (22:7982) 
[21] M. Raynaud, Schémas en groupes de type $(p, \ldots, p)$, Bull. Soc. math. France 102 (1974), 241-280. MR0419467 (54:7488)

[22] M. Reversat, Sur les revêtements de Schottky des courbes modulaires de Drinfeld, Arch. Math. 66 (1996), 378-387. MR1383902 (97e:11073)

[23] J-P. Serre, Trees, Springer, 1980. MR0607504 (82c:20083)

[24] M. van der Put, Discrete groups, Mumford curves and theta functions, Ann. Fac. Sci. Toulouse 1 (1992), 399-438. MR.1225666 (94h:14021)

[25] A. Weil, Basic number theory, 3rd edition, Springer-Verlag, 1974. MR0427267 (55:302)

Department of Mathematics, Stanford University, Stanford, California 94305

E-mail address: papikian@math.stanford.edu 